\title{
Escalado: Arquímedes y un divisor de voltajes
}

\author{
Scaling: Archimedes and a voltaje divider
}

\author{
C. H. Wörner*1
}

${ }^{1}$ Programa de Magister en Didáctica de las Ciencias Experimentales, Pontificia Universidad Católica de Valparaíso, Av.Brasil, 2950, Valparaíso, 2340000, Chile

Recibida en 12 de Junio, 2017. Aceptado en 07 de Julio, 2017

\begin{abstract}
Se aplica la técnica de escalonamiento en dos cuestiones, uno de ellas, una demostración geométrica de Arquímedes y el otro, un circuito atenuador. El contraste de las cuestiones abordadas ambos ejemplos, ilustra la unificación de técnicas en ambos casos.
\end{abstract}

Palabras clave: escalado, geometría griega, circuitos óhmicos.

Scaling techniques are applied to two simple cases. One of them is a Archimedes' geometrical proof and the other a damped circuit. The contrast between these two cases, shows the unity of the scaling technique.

Keywords: Scaling, Greek geometry, ohmic circuits.

Aparte del propio contenido de la ciencia que se enseña en la escuela, es necesario contar una especie de "chispa" que detone el interés de los alumnos por el tema que se trate. Así, Ausubel [1, 2] propone o que él denomina "organizadores previos" (advance organizers ). La actividad que se describe en esta nota puede ser entendida como una manera de proporcionar al profesor un nuevo elemento que puede ser usado en esta dirección. También merece ser usado como parte de un programa de clubes de ciencia para alumnos más avanzados. La combinación de un enfoque poco usual y la revisión de la historia de las ciencias, puede además ampliar el contexto cultural de la docencia.

Arquímedes, en el desarrollo de una de sus demostraciones para la cuadratura de la parábola [3] hace uso de la serie geométrica:

$$
S=(1 / 2)^{2}+(1 / 4)^{2}+(1 / 8)^{2}+(1 / 16)^{2}+\ldots
$$

Él encontró el valor de la serie con una demostración geométrica que revela su genio. Se dibuja un cuadrado de lado unidad (ver Fig. 1) y dentro de él se dibujan cuadrados de lados $\frac{1}{2} \frac{1}{4} \frac{1}{8}, \ldots$, como se muestra en la figura. De este modo el cuadrado unidad tiene su superficie trisectada (las dos figuras no coloradas que completan el cuadrado tienen la misma área que la superficie de colores azul y amarillo).

Así, simplemente:

$$
3\left[(1 / 2)^{2}+(1 / 4)^{2}+(1 / 8)^{2}+(1 / 16)^{2}+\ldots\right]=1
$$

y

$$
S=1 / 3
$$

Ahí termina la demostración de Arquímedes.

*Endereço de correspondência: carlos.worner@pucv.cl
De otra manera, es posible argumentar de una forma ligeramente diferente. En la figura los cuadrados amarillos son exactamente similares a la serie de cuadrados originales, sólo que escalados por un factor (lineal) de 1/2.De este modo la suma se puede escribir:

$$
S=(1 / 2)^{2}+(1 / 2)^{2}\left[(1 / 2)^{2}+(1 / 4)^{2}+\ldots\right]
$$

donde el factor que precede al paréntesis cuadrado es el factor de escala. Fácilmente:

$$
S=(1 / 2)^{2}(1+S)
$$

y finalmente,

$$
S=(1 / 3)
$$

que coincide con el resultado expresado en la Ec. 1.

Otra cuestión enteramente diferente puede ser resuelta usando el argumento del escalado. Enunciaremos el problema, ya propuesto en la literatura [4], como el estudio de un divisor de voltaje o atenuador compuesto por un conjunto infinito de resistencias óhmicas. Las resistencias son iguales, $R$, dispuestas como se muestra en la figura 2a. La pregunta es el cálculo de la resistencia equivalente de este sistema, es decir qué marcaría un óhmetro conectado entre los puntos $A$ y $B$.

La topología del circuito en estudio hace que el sistema esté escalado (de orden uno). Consideremos la serie infinita de resistencias $R$ situadas a la derecha de los puntos $C$ y $D$. Es fácil ver que ellas reproducen el sistema de resistencias original, tal como se muestra en la figura $2 \mathrm{~b}$. De este modo es fácil obtener la ecuación para el circuito utilizando las reglas de las conexiones serie-paralelo:

$$
R_{e q}^{2}-R R_{e q}-R^{2}=0
$$

La solución (positiva) de la ecuación anterior es 

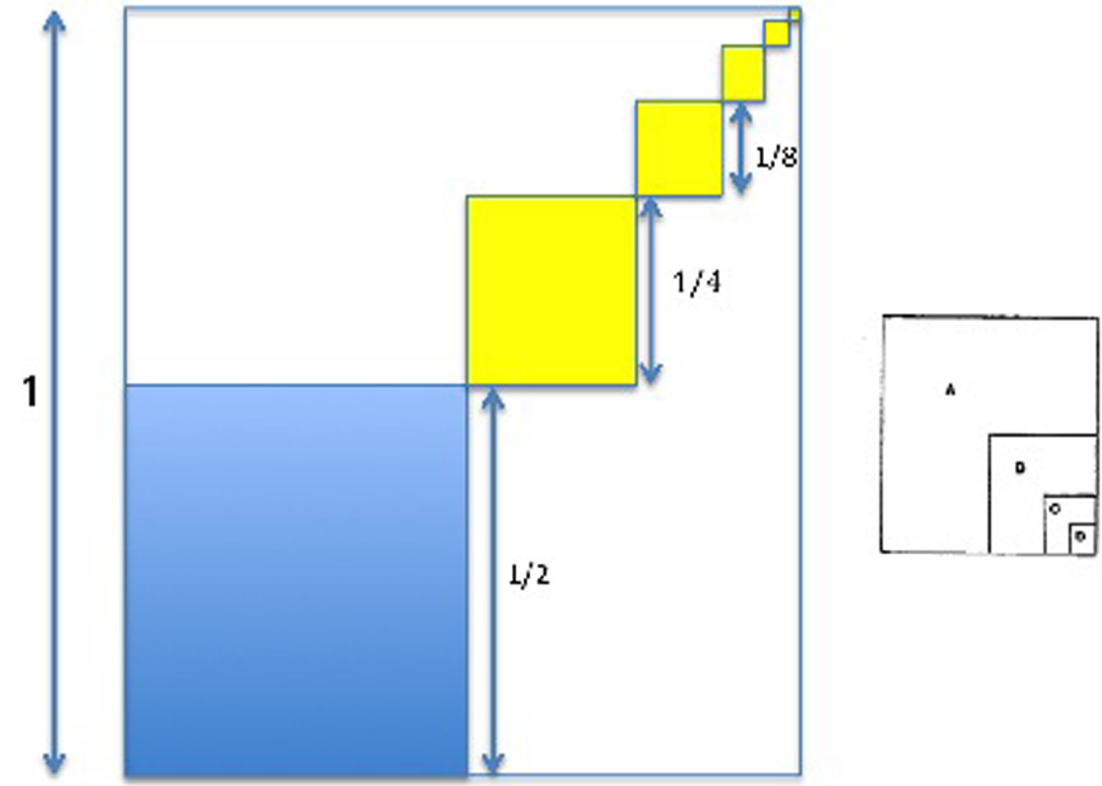

Figura 1: Construcción de Arquímedes. El inserto muestra el dibujo original de Arquímedes.
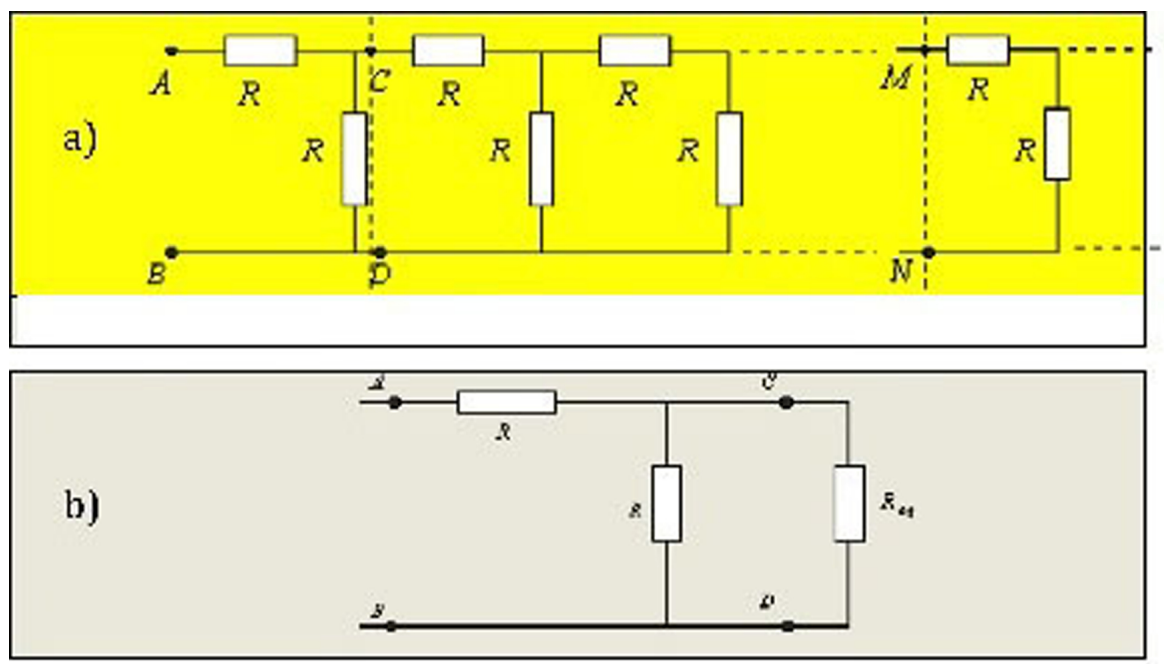

Figura 2: a)Divisor de voltaje con infinitas resistencia iguales $R$. b) circuito equivalente.

$$
R_{e q}=\phi R, \quad \text { donde } \quad \phi=\frac{1+\sqrt{5}}{2}
$$

es la razón áurea o divina [5].

Con este pequeño ejercicio, queremos mostrar la ubicuidad los método de escalado, que generalmente se desconocen en la instrucción elemental.

\section{Referencias}

[1] D.P. Ausubel, Journal of Educational Psychology 51, 267 (1960).

[2] D. Ausubel, Review of Educational Research 48, 251 (1978).
[3] Archimedes, The Quadrature of the Parabola, translated by T.L. Heath (Cambridge University Press, Cambridge, 1897).

[4] M. Alonso y E. Finn, Fundamental University Physics, (Addison-Wesley, Boston, 1967), v. 2; E.M. Purcell, Electricity and Magnetism (McGraw-Hill, New York, 1985), $2^{\mathrm{a}}$ ed. También ha sido considerado en la literatura especializada T.P Srinivasan, Am. J. Phys. 60, 461, 1992; B. Denardo, J. Earwood y V. Sazonova, Am. J. Phys. 67, 981, 1999.

[5] H.E. Huntley, The Divine Proportion (Dover Publications, Mineola, 1970). 